Glia. 2014 December ; 62(12): 2096-2109. doi:10.1002/glia.22729.

\title{
Interaction of $\mathrm{mTOR}$ and Erk1/2 signaling to regulate oligodendrocyte differentiation
}

\author{
JinXiang Dai, Kathryn K. Bercury, and Wendy B. Macklin \\ University of Colorado School of Medicine, Department of Cell and Developmental Biology, \\ 12801 E. $17^{\text {th }}$ Ave., Aurora, CO 80045
}

\begin{abstract}
A multitude of factors regulate oligodendrocyte differentiation and remyelination, and in order to elucidate the mechanisms underlying this process, we analyzed the interactions of known signaling pathways involved in these processes. Previous work from our lab and others shows that Akt, mTOR and Erk 1/2 are major signaling pathways regulating oligodendrocyte differentiation and myelination in vitro and in vivo. However, the relative contribution of the different pathways has been difficult to establish because the impact of inhibiting one pathway in in vitro cell culture models or in vivo may alter signaling through the other pathway. The current studies were undertaken to clarify the interactions between these major pathways and understand more specifically the crosstalk between them. Oligodendrocyte differentiation in vitro required Akt, mTOR and Erk 1/2 signaling, since inhibition of Akt, mTOR or Erk 1/2 resulted in a significant decrease of MBP mRNA and protein expression. Interestingly, while inhibition of the Erk1/2 pathway had little impact on Akt/mTOR signaling, inhibition of the Akt/mTOR pathways significantly increased Erk1/2 signaling, although not enough to overcome the loss of Akt/mTOR signaling in the regulation of oligodendrocyte differentiation. Furthermore, such crosstalk was also noted in an in vivo context, after mTOR inhibition by rapamycin treatment of perinatal pups.
\end{abstract}

\section{Keywords}

Akt; mTOR; Erk1/2; oligodendrocyte; myelin basic protein

\section{Introduction}

Oligodendrocyte differentiation is a highly regulated process. It is essential for nervous system development and for normal nervous system function in the adult. Multiple sclerosis (MS) is the major demyelinating disease of the central nervous system (CNS), and it results in loss of myelin and oligodendrocytes, which leads to serious neurological deficits. Oligodendrocyte progenitor cells (OPCs) are abundant in chronic MS lesion sites, which, along with other clinical observations, strongly indicates that inefficient remyelination in MS largely results from an inability of OPCs to differentiate to myelinating oligodendrocytes (Chang et al. 2000; Chang et al. 2002). Thus, identifying the cellular

Corresponding author: Wendy B. Macklin, Department of Cell and Developmental Biology, University of Colorado School of Medicine, 12801 E. $17^{\text {th }}$ Ave, Mail Stop 8108, Aurora, CO 80045, (303) 724-3426, Wendy.Macklin@ucdenver.edu. 
mechanisms that control the differentiation of OPCs through distinct stages of oligodendrocyte lineage progression may provide a therapeutic opportunity to promote remyelination in neurodegenerative diseases.

Although several extracellular signaling molecules and transcription factors are known to be essential for OPC differentiation (see (Mitew et al. 2013)), the intracellular signaling pathways that communicate between extracellular regulators and crucial transcription factors are poorly understood. In the past several years, we and others have established that the Akt/ mTOR (mammalian Target Of Rapamycin) and Erk (Extracellular Signal-regulated Kinase) $1 / 2$ pathways are critical for oligodendrocyte differentiation both in vitro and in vivo (Flores et al. 2000; Flores et al. 2008; Narayanan et al. 2009; Tyler et al. 2009; Fyffe-Maricich et al. 2011; Ishii et al. 2012; Guardiola-Diaz et al. 2012; Ishii et al. 2013). Previous in vitro studies on the role of specific signaling pathways in oligodendrocyte differentiation have focused on single pathways. However, studying signaling pathways individually ignores potential pathway interactions that can result in positive or negative effects of one pathway on another. In cancer research, multiple signaling pathways triggered by common upstream stimuli are often coordinately regulated by extensive crosstalk, and it is well established that pharmacological inhibition of the mTOR pathway can feedback to upregulate the MAPK (Mitogen Activating Protein Kinase) pathway through the S6K (p70 S6 kinase) -PI3K (Phosphatidyl inositol-3-kinase) -Ras signaling cascade modulated by IRS-1 (Insulin receptor substrate-1)(Carracedo et al. 2008b). In this signaling cascade, the PI3K/Akt/mTOR pathway is linked to the Ras/Raf/MEK/Erk pathway through IRS-1. Normally mTOR activation of S6K feeds back on IRS-1 to inhibit both PI3K and MAPK. Blocking mTOR with rapamycin results in loss of active S6K and of its negative feedback on IRS-1, leading to hyperactive IRS-1/PI3K, which feeds over to hyperactivate the Ras-Raf-MEK-Erk pathway. This interacting pathway is modeled in Figure 4.

The signaling pathways regulating oligodendrocyte differentiation are beginning to be understood, but crosstalk between such pathways will clearly impact this. Thus, we address the interactions of these two important signaling pathways that simultaneously regulate oligodendrocyte differentiation. In this study, we found that both major signaling pathways through Akt/mTOR and MEK/Erk 1/2 are necessary for full differentiation of OPCs to mature oligodendrocytes in vitro. Furthermore, combinatorial inhibition of the Akt/mTOR and Erk 1/2 pathways revealed the essential role of these signaling pathways in regulating myelin basic protein (MBP) gene and protein expression throughout oligodendrocyte maturation.

\section{Materials and Methods}

\section{Animals}

PLP-EGFP homozygous transgenic mice (Mallon et al. 2002), bred in house, were used in some immunohistochemical studies. Wild type C57B1/6 mice were obtained from JAX laboratories (Bar Harbor, ME) and were bred to generate embryonic mouse neurosphere cultures or for immunohistochemistry studies. Sprague Dawley rats (Harlan, Madison, WI) were bred to generate pups for the mixed glial cultures. 


\section{Reagents}

Cell culture reagents including DMEM, HBSS, Pen-Strep, L-Glutamine and N2 supplement were obtained from Invitrogen Life Technologies (Carlsbad, CA). Hydrocortisone (\#H6909), Biotin (\#B4639), triiodothyronine (\#T6397), poly-D-lysine (\#P0899) and laminin (\#L2020) were obtained from Sigma-Aldrich (St Louis, MO). Fibronectin (\#FC010) was purchased from Millipore (Billerica, MA). Recombinant human FGF-basic (\#100-18B) and PDGF-AA (\#100-13A) were acquired from PeproTech (Rocky Hill, NJ). Rapamycin (\#1292) and LY294002 (\#1130) were purchased from TOCRIS (Minneapolis, MN) and U0126 (\#9903) was obtained from Cell Signaling Technology (CST) (Beverly, MA). Torin1 (1 $\mathrm{mM}$ stock) was a kind gift of Dr. David Sabatini (Whitehead, MIT, Cambridge, MA). Antibodies: mouse myelin basic protein [SMI-94], Covance (Princeton, NJ), mouse betaTubulin \#T8328 Sigma Aldrich (St. Louis, MO), rabbit myelin basic protein \#ab980 Millipore (Billerica, MA) and goat Sox10 [N20] \#Sc-17342 Santa Cruz Biotechnology, Inc. (Santa Cruz, CA). All antibodies for signaling pathways were obtained from Cell Signaling Technology (Beverly, MA): p-p44/42 MAPK-T202/Y204 XP (\#4370), pan-Erk1/2 (\#4696), p-Akt-T308 (\#2965), p-Akt-S473 XP (\#4060), pan-Akt (\#2920), p-S6 ribosomal protein(\#2215), p-mTOR-S2448 XP(\#5536), p-mTOR-S2448 immunohistochemistryspecific (\#2976), total mTOR (\#2972), GAPDH (\#2118) and Insulin Receptor Substrate Antibody Sampler Kit (\#3015). Rabbit Olig2 antibody was a kind gift from Dr. Charles Stiles (Dana Farber Cancer Institute, Boston, MA). O4 and O1 hybridomas were obtained from the late Dr. Steven Pfeiffer (UConn Health Science Center, Framington, CT).

\section{Oligodendrocyte rat and mouse progenitor cell cultures}

All animal experimentation protocols were approved by the University of Colorado School of Medicine Animal Care and Use Committee (IACUC) and were conducted in accordance with the National Institutes of Health guidelines for the care and use of laboratory animals. Mixed glial cultures were generated from 0d to 3d old Sprague Dawley rat pups as described previously (Gudz et al. 2006). The cerebra of rat pups were dissected, and mechanically dissociated to generate a single-cell suspension. Cells were plated into poly-D-lysine (PDL)coated $75 \mathrm{~cm}^{2}$ flasks and grown in DMEM high glucose with $10 \%$ fetal bovine serum (FBS) for 10-12 days. OPCs were purified from mixed glial cells by a shake-off procedure (McCarthy and de Vellis 1980). OPCs from mouse neurospheres were generated with adaptions to the protocol from (Pedraza et al. 2008). Purified rat or mouse OPCs were seeded on PDL/fibronectin/laminin-coated dishes or $12 \mathrm{~mm}$ coverslips in DMEM supplemented with $\mathrm{N} 2$ media plus FGF/PDGF (both at $10 \mathrm{ng} / \mathrm{ml}$ ). Cells were allowed to recovery for $48 \mathrm{~h}$. To initiate differentiation, cells were shifted to mitogen free N2 media plus $40 \mathrm{ng} / \mathrm{ml}$ triiodothyronine (T3) (Tokumoto et al. 1999). Kinase inhibitors were suspended in DMSO (1000x) and diluted into differentiation media at the indicated concentrations. Differentiation media plus or minus kinase inhibitors was replenished every other day.

\section{Immunocytochemistry and in vitro quantification}

After 1, 3, or $5 \mathrm{~d}$ of OPC differentiation plus/minus kinase inhibitors, coverslips were fixed with $4 \%$ paraformaldehyde for $15 \mathrm{~min}$ at RT and permeabilized with $0.1 \%$ Triton X-100 for 
10 min. After blocking with 3\% BSA in PBS for 60 min at RT, cells were incubated with primary antibodies overnight at $4{ }^{\circ} \mathrm{C}$. For double labeling, $\mathrm{O} 1$ or $\mathrm{O} 4$ primary antibodies were added to live cells and incubated for 1 hour before coverslips were fixed and immunohistochemistry was performed with other primary antibodies listed above. Secondary antibodies were added for 1 hour at room temp and coverslipped with Fluoromount F (\#0100-01; Southern Biotech, Birmingham, AL). The number of O4-, O1- or MBP-positive cells present was quantified on a Zeiss AxioImager M2 microscope. Images were acquired with a 10x objective; three fields per coverslip were imaged for two coverslips per condition in each experiment. The total number of cells counted per condition averaged 700-1500 for each experiment.

\section{Western Blot Analysis}

After treatment, cells were rinsed twice with ice cold PBS, and cell lysates were made in RIPA buffer: $25 \mathrm{mM}$ Tris-HCl (pH 7.5), $150 \mathrm{mM} \mathrm{NaCl} ; 1$ mM EDTA, $1 \%$ NP-40, 0.1\% sodium deoxycholate, supplemented with complete mini protease inhibitor cocktail (Roche \#04693159001 Indianapolis, IN) and phosphatase inhibitor cocktail set II (Calbiochem \#564652, San Diego, CA). After $10 \mathrm{~min}$ on ice, the lysates were sonicated and stored at $-20^{\circ} \mathrm{C}$. Protein was quantified with the DC ${ }^{\mathrm{TM}}$ protein assay (Bio-Rad\#500-011, Berkeley, CA). Samples were electrophoresed on SDS polyacrylamide gels and blotted. Membranes were blocked in 5\% BSA for $1 \mathrm{~h}$. Primary antibodies were diluted 1:1000 in 5\% BSA and incubated with the membranes at $4^{\circ} \mathrm{C}$ overnight. Protein bands were detected with an Odyssey infrared scanner (LI-COR, Lincoln, NE) with goat anti-rabbit IRDye 680RD and goat anti-mouse IRDye $800 \mathrm{CW}$ secondary antibodies $(1: 15,000)$. Protein expression was quantified using Odyssey scanner software 2.0.

\section{Immunohistochemistry}

Perfusion of mice and immunostaining for myelin proteins were performed as described (Trapp et al. 1997) with slight modifications. Immunohistochemistry for phosphorylated antibodies was done using the protocol of Narayanan et al. (2009), with some modifications. Phospho-S6 ribosomal protein antibody was used to test section quality for all samples. Only sections with robust and uniform phospho-S6 ribosomal protein signal in the neocortex were chosen for further staining with other antibodies. On day 1, brains were sectioned at 30 $\mu \mathrm{m}$ on a sliding microtome. Free-floating sections were washed three times in PBS and antigen retrieval was performed with $10 \mathrm{mM}$ sodium citrate $(\mathrm{pH} 6.0)$ at $65^{\circ} \mathrm{C}$ for $10 \mathrm{~min}$ in a BioWave (Ted Pella). Sections were rinsed in PBS twice, blocked in blocking solution (200 $\mathrm{mM} \mathrm{NaCl}, 50 \mathrm{mM}$ Tris. $\mathrm{HCl}, 100 \mathrm{mM}$ L-lysine, $150 \mathrm{mM}$ glycine) with 5\% normal donkey serum (NDS) and 3\% Triton X-100 for $1 \mathrm{~h}$ at RT and incubated in primary antibodies in 3\% NDS containing $1 \%$ Triton X-100 for $72 \mathrm{~h}$ at $4^{\circ} \mathrm{C}$. All phospho-antibodies and Sox 10 were used at 1:200. On day 4, sections were washed three times in PBS, incubated with secondary antibodies (1:800) at RT, then counter stained for DAPI for $5 \mathrm{~min}$. Finally, sections were washed three times in PBS and mounted in Vectashield (Vector Laboratories).

\section{RNA extraction and quantitative real-time PCR}

RNA isolation and real-time PCR were performed as described (Pedraza et al. 2008). For RNA isolation, cells were seeded onto PDL/Fibronectin/Laminin-coated $10 \mathrm{~cm}$ dishes. After 
5 days treatment, cells were washed twice with cold PBS, $1 \mathrm{ml}$ of Trizol reagent (Invitrogen) was added, and the plate was incubated on ice for $10 \mathrm{~min}$. The cells in the Trizol reagent were then collected and RNA was extracted in phenol/chloroform. cDNA was generated from $1 \mu \mathrm{g}$ total RNA by Superscript II (Invitrogen) reaction and transcripts were detected and amplified by qRT-PCR in a Light Cycler machine (Roche, Indianapolis, IN), following the manufacturer's instructions.

\section{Rapamycin injections}

Intraperitoneal (IP) injections of rapamycin were performed as previously described (Narayanan et al. 2009). Rapamycin (LC lab\#R-5000, Woburn, MA) was dissolved in 100\% ethanol and stored at $-20^{\circ} \mathrm{C}$ until use. Immediately before injection, rapamycin was diluted in 5\% PEG-400, 5\% Tween 80, and 4\% ethanol. Postnatal day 7 (P7) mice were injected with rapamycin $(10 \mathrm{mg} / \mathrm{kg}$ body weight) or vehicle. $24 \mathrm{~h}$ later mice were perfused, tissue was dissected and post-fixed with $4 \%$ paraformaldehyde and analyzed by immunohistochemistry as described above.

\section{Statistical Analysis}

Statistics were performed using one or two way ANOVA statistical testing with Tukey's post-hoc correction or student's t-test with False Discovery Rate (FDR) correction with FDR $=0.05$, depending on the data set analyzed. Statistics were calculated using GraphPad Prism (La Jolla, CA) and graphed using Origin 8 software (Northampton, MA). Error bars represent Standard Error of the Mean (SEM).

\section{Results}

\section{Activation of the Akt/mTOR and Erk 1/2 pathways correlated with OPC differentiation in vitro}

Both the PI3K/Akt/mTOR and Ras/MEK/Erk 1/2 pathways are involved in OPC proliferation, survival and migration in response to various growth factors as oligodendrocytes mature (see (Mitew et al. 2013) for review). Recently, the PI3K/Akt/ mTOR and Ras/MEK/Erk 1/2 pathways have also been shown to regulate oligodendrocyte differentiation at distinct stages in vitro (Tyler et al. 2009; Guardiola-Diaz et al. 2012). The current studies were developed to investigate the interactions of these two pathways. Initial western blot studies set the baseline of signaling through both the Akt/mTOR and Erk 1/2 signaling pathways during normal oligodendrocyte differentiation in vitro. Thus, rat OPCs were differentiated in vitro by removal of PDGF/FGF and addition of T3, and samples were taken 10 min to 5 days later. Full activation of Akt is dependent on the phosphorylation of serine 473 (S473) by mTORC2 and threonine 308 (T308) by phosphoinositol-dependent kinase 1 (PDK1). Phosphorylation at both sites of Akt and of Erk1/2 increased during oligodendrocyte maturation after mitogen withdrawal, which correlated with significant increases in MBP protein expression from day 1 to 5 (Fig. 1A, B). Phosphorylation of mTOR and the known downstream target of mTORC1, pS6 ribosomal protein (pS6RP), did not significantly change during OPC maturation in vitro (Fig. 1A, B). Since Akt, mTOR and Erk1/2 can share the common upstream regulator IRS-1, we analyzed IRS-1 expression and found that the major inhibitory phosphorylation sites on IRS-1 (pIRS-1 S307, pIRS-1 S318 
and pIRS-1 S612) decreased as oligodendrocytes differentiated. These inhibitory phosphorylation sites on IRS-1 generally induce turnover of IRS-1 (Mothe and Van Obberghen 1996; De Fea and Roth 1997; Delahaye et al. 1998; Li et al. 1999), which can be seen from day 1 , and, as expected, the decrease in inhibitory IRS-1 phosphorylation was inversely correlated with pAkt and pErk1/2 increases. In multiple experiments, we also observed a striking band at pIRS-1 S612 at 2d when there were also transient decreases of pAkt and pErk 1/2 signaling, but the functional consequence of the transient decrease of both Akt and Erk1/2 signaling at $2 \mathrm{~d}$ is unclear at this point. The data in Figure 1 indicated that both Akt and Erk 1/2 were activated during OPC differentiation, and that during early differentiation, they might be coordinately regulated through IRS-1. Intriguingly, mouse OPCs obtained from embryonic neurospheres failed to fully differentiate under these conditions, and western blot analyses indicated that these pathways were not fully activated in these cells (supplement Fig. 1). These data further support the concept that both Akt and Erk1/2 signaling are involved in oligodendrocyte differentiation.

\section{Inhibition of Akt/mTOR and Erk 1/2 pathways impacted OPC differentiation in vitro}

In order to assess the relative contribution of Akt/mTOR and/or Erk 1/2 to oligodendrocyte differentiation in vitro, we treated cells with kinase inhibitors for both pathways. LY294002, an inhibitor of PI3K, blocks the downstream activation of Akt and mTOR signaling. mTOR and Erk are inhibited by rapamycin and U0126, respectively. mTOR signaling is mediated through two complexes, mTORC1 and mTORC2, and mTORC1 is classically defined as the rapamycin target, although at high doses or after chronic exposure, rapamycin can also inhibit mTORC2 (Sarbassov et al. 2006; Thoreen and Sabatini 2009). In these studies, however, rapamycin (1 uM) blocked only mTORC1 and not mTORC2 signaling, as demonstrated by western blot analysis of their respective downstream targets, pS6RP or pAkt 473 (Figure 4A). Therefore, in order to effectively inhibit both mTOR complexes, we used Torin1 (Liu et al. 2010). Previous studies have shown that rapamycin or U0126 treatment alone blocks the early stages of OPC differentiation in vitro (Tyler et al. 2009; Guardiola-Diaz et al. 2012). Our studies were designed to investigate the effect(s) of single or combinatorial treatment with LY294002, rapamycin, Torin1 or U0126 to investigate the interactions of these pathways.

Inhibitors targeting the different pathways were added when OPCs were switched to differentiation media, and differentiation was defined in these studies by MBP expression. We tested all inhibitors in dose response analysis for their specificity and efficiency and their impact on cell survival over the 5 days of differentiation (not shown) and optimal conditions were chosen. Over 5 days exposure to U0126 or rapamycin, approximately 15\% of cells were lost (Supplemental Figure 2). This likely resulted from cell death rather than reduced proliferation, since mitogens were removed from the media to induce differentiation. By contrast, Torin1 exposure reduced cell number by approximately $63 \%$ relative to DMSO control, likely through inducing cell death, since Torin 1 has been shown in other systems to mediate cell death via apoptosis (Francipane and Lagasse 2013; Volkers et al. 2013). 
As expected, in DMSO-treated control cells, there was high MBP protein expression after 5 days (Fig. 2A). We also assessed Olig2 protein expression as an additional oligodendrocytespecific control; the level of Olig2 was constant and consistent with the $\beta$-tubulin level. U0126 or rapamycin treatment alone for $5 \mathrm{~d}$ decreased MBP expression by $42 \%$ or $71 \%$, respectively (Fig. 2B). (These doses of rapamycin $(1 \mu \mathrm{M})$ or U0126 $(20 \mu \mathrm{M})$ were shown to block mTORC1 (p-S6 RP) or Erk 1/2 (p-Erk1/2) activation, respectively [Fig. 4A].) There was a more dramatic effect of LY294002 $(25 \mu \mathrm{M})$ or Torin1 $(250 \mathrm{nM})$, with MBP expression reduced by $88 \%$ or $92 \%$ respectively.

In other studies, the impact of the different inhibitors on oligodendrocyte differentiation was calculated as the percentage of total Olig2+ cells that expressed O4, O1 or MBP. Treatment of cells with U0126 (blocking Erk 1/2), Rapamycin (blocking mTORC1), or Torin1 (blocking mTORC1 and mTORC1/2) for $1 \mathrm{~d}$ had little impact on the percent that differentiated to $\mathrm{O} 4+$ cells. Treatment for 3 days or 5 days with U0126 or rapamycin resulted in a comparable reduction in the percent of Olig2+ cells that differentiated to O1+ cells or MBP+ cells, respectively. Thus, 5 days treatment reduced the number of MBPpositive cells by $59 \%$ and $61 \%$ respectively, relative to controls (Fig. 2C, D, E). These data suggested that Erk 1/2 or mTORC1 inhibition had comparable impact on the late progenitor (O4+) to immature oligodendrocyte (O1+) transition, blocking $~ 60 \%$ of differentiation at that stage. On the other hand, the inhibition of mTORC 1 and mTORC 2 signaling by Torin 1 essentially prevented cell differentiation even to the $\mathrm{O} 1+$ stage.

\section{Akt/mTOR and Erk 1/2 pathways regulate distinct stages of oligodendrocyte differentiation in vitro}

In order to more specifically define the time frame in which Akt/mTOR and Erk activation promoted oligodendrocyte differentiation, we treated cells with inhibitors, starting at multiple time points after the shift to differentiation medium. Thus, we started treatment at $0,1,2,3$ or 4 days post addition of differentiation medium. On day 5, MBP protein expression was quantified by western blot analysis and compared with DMSO-treated control cultures (Fig. 3). Consistent with the previous experiments, increased MBP protein expression was seen by $5 \mathrm{~d}$ differentiation in the DMSO-treated controls, whereas the MBP level in cells treated with LY294002, rapamycin or Torin1 for $5 \mathrm{~d}$ was less than $\sim 10 \%$ of control, and cells treated with U0126 had approximately 50\% of control MBP (Fig. 3). These data indicated that U0126 did not as dramatically reduce MBP protein expression, compared to some of the other inhibitors, although it must be noted that other immunohistochemical studies of U0126 treated oligodendrocytes have shown a greater impact on differentiation than we saw in these studies (Guardiola-Diaz et al. 2012). Cells allowed to differentiate for 1 or 2 days and then treated with Torin 1 expressed $11 \%$ or $34 \%$ of MBP respectively, compared with DMSO-treated cells (Fig. 3D). A similar but less significant impact on MBP protein expression was also found in the LY294002- or rapamycin-treated cells (Fig. 3B, D). Thus, rapamycin treatment had its greatest impact on MBP protein levels 1d after differentiation, but when added from 2-4 days, MBP protein levels were comparable to DMSO controls (Fig. 3D). Similarly, when Torin1 was added to the cells $3 \mathrm{~d}$ and $4 \mathrm{~d}$ after differentiation, the MBP expression level was not significantly different from DMSO-treated cells after 5d of differentiation (Fig. 3D). These data indicated 
that $\mathrm{mTORC} 1$ and/or mTORC 2 were critical for the early transition from late progenitor to the immature oligodendrocyte at $1 \mathrm{~d}$ post addition of differentiation media, but less important at later stages. This is consistent with the study of (Tyler et al. 2009), which showed that rapamycin greatly inhibited the initial differentiation of the progenitor cell to the immature oligodendrocyte, but had much less effect if added to oligodendrocytes after that initial differentiation stage. By contrast, Erk 1/2 inhibition at any time point reduced the expression of MBP by 30-40\% compared with that of DMSO-treated cells after $5 \mathrm{~d}$ of differentiation suggesting that although Erk 1/2 inhibition did not as dramatically reduce the amount of MBP protein per oligodendrocyte compared to other inhibitor treatments, loss of Erk1/2 activity even at late stages reduced MBP expression (Fig. 3B).

\section{The crosstalk between the Akt/mTOR and Erk 1/2 pathways was not bidirectional}

Since the Akt and Erk 1/2 pathways were both activated at the initiation of differentiation, it appeared likely that they might be coordinately regulated in response to differentiation stimuli. As noted above, in other cell systems there is functional crosstalk between the Akt/ mTOR and Erk 1/2 pathways. This crosstalk results from downregulation of pS6RP, which reduces the inhibitory impact of IRS-1 on signaling through Erk 1/2. In order to test potential crosstalk between these pathways in differentiating oligodendrocytes, the pathwayspecific inhibitors were added alone or in combination to OPC cultures at the onset of differentiation in vitro. Erk 1/2 inhibition by U0126 had little effect on Akt or mTOR activities (Fig. 4A). By contrast, inhibition of multiple parts of the Akt/mTOR pathway had an intriguing impact on Erk 1/2 phosphorylation. LY294002 treatment clearly reduced Akt phosphorylation and also increased Erk 1/2 phosphorylation, compared with DMSO-treated cells, demonstrating crosstalk between the Akt and Erk 1/2 pathways. Additionally, a striking elevation of phosphorylated Erk $1 / 2$ was observed in oligodendrocytes treated with rapamycin or with Torin 1 (Fig. 4A). In order to test whether mTOR inhibition could upregulate Erk 1/2 activation through an S6K1-PI3K dependent feedback loop, we treated cells with LY294002 and rapamycin in combination, which would block PI3K activity and phosphorylation of S6K1 (Harrington et al. 2005). Indeed, PI3K inhibition by LY294002 reduced the mTORC1 inhibition-mediated Erk $1 / 2$ activation in differentiating oligodendrocytes (Fig 4A, top row). In contrast, the Torin1-mediated Erk 1/2 pathway activation actually increased in the presence of LY294002, which indicated that mTORC2 may feedback to upregulate the Erk 1/2 pathway through a PI3K-independent mechanism.

Possible mechanisms of this crosstalk between the Akt/mTOR and Erk 1/2 pathway have been modeled (Fig. 4B). Under normal conditions, there is a balanced relationship between the Akt/mTOR and Erk 1/2 pathways. Upon inhibition of Akt/mTOR (Figure 4Bb), S6K activity is reduced, and it no longer feeds back on IRS-1 releasing the inhibition of Erk 1/2 by IRS-1 resulting in increased phosphorylation of Erk 1/2. The most dramatic increase in Erk 1/2 activation was seen when Akt, mTORC1 and mTORC2 were inhibited in combination (Figure 4Be), suggesting an additional normally negative regulation at the $\mathrm{PI} 3 \mathrm{~K} / \mathrm{Akt} / \mathrm{mTOR}$ level on that pathway. Of most interest, the crosstalk between these two pathways in oligodendrocytes appeared to be unidirectional. Thus the Akt/mTOR pathway appeared to normally have a negative impact on Erk1/2 activity, and that negative regulation 
was reduced upon Akt/mTOR inhibition. On the other hand, when Erk 1/2 was inhibited, there was no change in Akt/mTOR phosphorylation.

The Akt, mTOR and Erk 1/2 signaling pathways regulated both MBP mRNA and protein expression in vitro

The crosstalk between the Akt/mTOR and Erk 1/2 pathways raised the possibility that the Akt/mTOR and Erk 1/2 pathways could integrate different signals to regulate the common downstream targets that are essential for oligodendrocyte differentiation. Because high concentrations of the PI3K inhibitor (LY294002) and mTORC 1/2 inhibitor (Torin1) fully blocked oligodendrocyte maturation, reduced concentrations of LY294002 and Torin1 were used to assess their combinatorial impact on MBP mRNA and protein expression with Erk1/2 inhibitor U0126. At the onset of oligodendrocyte differentiation, pathway-specific inhibitors were added to OPC cultures individually or in combination. After $5 \mathrm{~d}$ differentiation, MBP mRNA or protein expression was detected by RT-qPCR (Fig. 5A) or western blot (Fig. 5B). MBP mRNA and protein expression were reduced in single kinase inhibitor treatments of the oligodendrocyte cultures (Fig. 5A, B), although because of the reduced LY294002 or Torin1 concentration, the inhibition was not as great as in Figure 2A. In this experiment the impact of PI3K inhibition (LY294002) on MBP mRNA expression was not statistically significant, due to the low concentration used, and there was no synergistic impact of combinatorial PI3K (LY294002) and Erk1/2 (U0126) inhibition on MBP mRNA expression, i.e., no greater impact than that of U0126. However, there was a clear synergistic effect of Erk1/2 inhibition in combination with mTORC1 or mTORC1/2 inhibition (Rapamycin + U0126 or Torin $1+$ U0126, Fig. 5A) to dramatically reduce MBP RNA expression.

In order to investigate whether there might be an additional impact of these signaling pathways on MBP protein translation, protein expression was analyzed by western blot (Fig. 5B). MBP protein in cells singly treated with rapamycin, Torin1, LY294002, or U0126 for 5 days was reduced to $19 \%, 57 \%, 26 \%$ or $23 \%$ of control (Fig. 5C). Given the relatively minimal effect of this concentration of LY294002 on MBP RNA expression (Fig. 5A), it was quite striking that MBP protein was dramatically reduced, suggesting that PI3K activity had a direct effect on MBP protein translation. By contrast, Erk1/2 activity appeared to affect primarily MBP RNA expression, since there was no greater loss of MBP protein than MBP RNA in the presence of U0126. However, there was an almost complete loss of MBP protein expression when oligodendrocytes were treated with U0126 in combination with LY294002, rapamycin or Torin1. The loss of MBP in the U0126/rapamycin or U0125/ Torin1 treated cells could be explained by the dramatic loss of MBP mRNA, but as noted above, clearly the U0126 treatment increased the translational impact of PI3K inhibition with LY294002.

\section{In vivo, Akt, mTOR and Erk $1 / 2$ signaling are dynamically regulated during oligodendrocyte maturation from P1 to P10}

Pharmacological inhibition of the Akt/mTOR and Erk1/2 pathways blocked oligodendrocyte differentiation at distinct stages in vitro and significantly reduced MBP mRNA and protein expression. However, it has been shown that protein kinase activities in vitro do not 
necessarily correlate to their role in oligodendrocytes in vivo (Flores et al. 2000; Flores et al. 2008; Guardiola-Diaz et al. 2012; Ishii et al. 2012). In order to determine the expression pattern and activity of the Akt, mTOR and Erk 1/2 pathways in vivo, we analyzed expression of the activated proteins in oligodendrocytes in the developing mouse brain corpus callosum at postnatal (P)1, P4, P7 and P10. These time points represent distinct stages of oligodendrocyte differentiation from progenitor cells to premyelinating cells to myelinating oligodendrocytes (Fig. 6). In line with the positive role of the Akt, mTOR and Erk 1/2 pathways in oligodendrocyte lineage progression from late progenitor cells to immature oligodendrocytes in vitro (Fig. 2E), we found peak expression of activated Akt, mTOR and Erk 1/2 in midline corpus callosum at P7 when OPCs were beginning to differentiate into premyelinating oligodendrocytes. Interestingly, pErk1/2 was initially expressed in the cytoplasm of OPCs at P1. It then shifted from cytoplasmic to nuclear localization between P4 and P7 in this region. Peak mTORC1 activity (as measured by phosphorylated S6 ribosomal protein) and mTORC2 activity (phosphorylated Akt S473) were also seen at this same time point, P7. Interestingly, these signaling pathways were all down-regulated in oligodendrocytes in the corpus callosum by $\mathrm{P} 10$, during the active transition from premyelinating to myelinating oligodendrocytes (Fig. 6B, D).

\section{Pharmacological inhibition of mTORC1 in vivo elevated the level of phosphorylated Erk $1 / 2$ signaling in oligodendrocytes}

The observation that the peak of activation of Akt, mTOR and Erk 1/2 in oligodendrocytes was in the P7 corpus callosum was consistent with our in vitro finding that Akt, mTOR and Erk $1 / 2$ were all coordinately activated as OPCs differentiated. This also raised the question of whether these kinases were coordinately regulated in response to differentiation stimuli in the in vivo context. To test this, P7 mice were given IP injections of rapamycin. Twenty-four hours post injection, brain tissue was collected, and expression of phosphorylated S6 ribosomal protein and phosphorylated Erk1/2 was analyzed (Fig. 7A). As expected, in the rapamycin-injected mice, there was a complete loss of phosphorylated S6 protein in corpus callosum oligodendrocytes, compared to vehicle-treated animals (Fig. 7A, upper right panel). In adjacent sections of the same rapamycin-injected P7 animals, pErk 1/2 signaling appeared elevated in oligodendrocytes in the corpus callosum (Fig. 7A, lower right panel). The number of pErk1/2-positive/Sox 10 positive oligodendrocytes was significantly increased in the rapamycin-injected mice compared to controls (39.4\% $\pm 2.4 \%$ vs $19.8 \%$ $\pm 2.9 \%$; Fig 7B). These data suggested that, as in vitro, there is crosstalk between Akt/mTOR and Erk 1/2 pathways in vivo.

\section{Discussion}

The current studies were undertaken to clarify the interactions of the Akt/mTOR and Erk 1/2 signaling pathways in regulating oligodendrocyte differentiation. Previous studies have arrived at different conclusions as to the relative contribution of each pathway to oligodendrocyte differentiation in vitro, based on inhibition of an individual pathway. Our data are consistent with earlier reports demonstrating that Akt/mTOR and Erk 1/2 are both necessary for oligodendrocyte maturation in vitro (Tyler et al. 2009; Guardiola-Diaz et al. 2012). However, the current studies also showed that these two pathways actually interact 
together to regulate oligodendrocyte maturation. Interestingly, inhibition of the Akt/mTOR pathway resulted in increased activation of Erk 1/2 signaling in differentiating oligodendrocytes, but not vice versa. However, the apparent compensatory over-activation of Erk1/2 was insufficient to allow oligodendrocyte differentiation, which remained blocked.

This in vitro model allowed us to predict an integrating mechanism involving IRS-1, which may regulate oligodendrocyte differentiation and myelination through both Akt/mTOR and Erk 1/2. Numerous studies have shown that decreased Akt/mTOR activation results in dysregulation of IRS-1 and activation of Erk 1/2 signaling (Harrington et al. 2005; Carracedo et al. 2008a; Carracedo et al. 2008b; Wang et al. 2013; Niba et al. 2013). Our data support this mechanism, and provide novel insight into the intrinsic signaling that could regulate proper spatial and temporal control of oligodendrocyte differentiation.

Interestingly, this study showed differences in kinase signaling in oligodendrocytes purified from rat mixed glia (Fig. 1) relative to mouse embryonic neurospheres (Supplemental Fig. 1). OPCs derived from mouse embryonic neurospheres failed to fully mature and express MBP, and also did not have the increases in Akt, mTOR and Erk 1/2 signaling seen in rat oligodendrocytes. While this could be a species difference, numerous investigators have studied mouse oligodendrocyte differentiation in vitro (Hennen et al. 2013; Silbereis et al. 2014), suggesting this is likely some aspect of our culture conditions. It may result from the maturation state of the progenitors, i.e., our mouse oligodendrocyte progenitor cells were from embryonic tissue, E14.5 cerebra, while the rat oligodendrocyte preparation was from postnatal tissue grown as mixed glia for ten days. Irrespective of why the mouse cells failed to fully differentiate under our growth conditions, the concomitant absence of long-term signaling through these pathways is correlative support for a role of these kinases in oligodendrocyte differentiation.

Many of the studies on crosstalk of these pathways identify both activating and inhibiting interactions between these two pathways. It is intriguing that in oligodendrocytes, the major crosstalk between the two pathways results in Akt/mTOR negatively regulating the Erk 1/2 pathway, but not vice versa. In other cells, the Ras/Erk pathway positively upregulates $\mathrm{PI} 3 \mathrm{~K} / \mathrm{Akt} / \mathrm{mTOR}$ signaling directly and indirectly. Purified Ras-GTP can directly bind purified PI3K and can activate it in PC12 cells (Rodriguez-Viciana et al. 1994).

Additionally, Erk can signal to inhibit the TSC1/2 complex, thereby releasing its inhibition of RHEB, allowing activation of mTOR (Ma et al. 2005). Thus, clearly in some cells, Erk1/2 signaling can impact the Akt/mTOR pathway, but it does not in oligodendrocytes in vitro. This might relate to their relative roles in myelination. Recent studies in the role of Erk1/2 in myelination suggest that although it has an impact in vitro (Guardiola-Diaz et al. 2012), it does not regulate oligodendrocyte differentiation in vivo, but it does regulate myelin thickness (Ishii et al. 2012). It is possible that the timing of the activities of the two different pathways affects their impact on the other pathway. If the Akt/mTOR pathway plays a role earlier than Erk1/2 in the oligodendrocyte development program, it is possible that their interactions are temporally and thus functionally separated. One could also speculate that this might explain why the increase in Erk1/2 activation upon Akt/mTOR inhibition cannot overcome the effect of the Akt/mTOR inhibition on oligodendrocyte differentiation. It is 
possible that $\mathrm{Akt} / \mathrm{mTOR}$ activation is essential for the eventual role the Erk1/2 signaling may play, and without that initial Akt/mTOR activation, Erk1/2 signaling is ineffectual.

In vivo, the peak activation of the Akt, mTOR and Erk 1/2 kinases in corpus callosum was at $\mathrm{P} 7$, in line with the positive role of these pathways in regulating early oligodendrocyte differentiation in vitro. Furthermore, when mTOR signaling was completely blocked by rapamycin in vivo, a significant increase of pErk1/2 signaling was observed (Fig. 7A, B) as seen in vitro. Additional in vivo work from our lab and others have recently shown that mTOR or mTORC1 (Raptor) deletion in oligodendrocytes delayed differentiation in the developing spinal cord and resulted in permanent reduction in myelin (Bercury et al. 2014; Wahl et al. 2014). However, in the brain, there was very little impact of mTOR or mTORC1 loss on oligodendrocyte differentiation, which was unexpected, given previous in vitro studies in which mTOR inhibition blocked brain-derived oligodendrocyte differentiation (Tyler et al. 2009). These apparently different findings in vivo relative to in vitro raise the question of why oligodendrocytes in vitro are more sensitive to the loss of individual signaling kinases. The in vivo data showing that eliminating a single signaling pathway may have minimal impact on oligodendrocyte differentiation and myelination in the brain suggest that compensatory mechanisms likely exist for the loss of that pathway, which may be compromised in vitro. Clearly, growing glial cells in culture changes their behavior. These cells differentiate, but they do not make myelin, and are responding to a vastly different and less complex environment than in vivo. Perhaps the absence of other cell types that are normally present in the in vivo context increases the impact of pharmacologic inhibition of isolated oligodendrocytes in vitro. Myelination is such an essential neurodevelopmental component that such cell-cell interactions in vivo might compensate for some detrimental changes in oligodendrocytes.

In conclusion, our studies provide evidence that different signaling pathways may contribute to the regulation of oligodendrocyte differentiation and myelination through crosstalk of multiple kinase pathways both in vivo and in vitro. Most notably, our data suggest that oligodendrocytes may attempt to overcome inhibition of Akt/mTOR by increases in $\mathrm{pErk}$ $1 / 2$ activity, but not vice versa. These studies emphasize the complexities of signaling crosstalk regulating oligodendrocyte differentiation and myelination.

\section{Supplementary Material}

Refer to Web version on PubMed Central for supplementary material.

\section{Acknowledgments}

The authors would like to thank Cecelia Culp for assistance editing the manuscript and Randall Wong for technical assistance with qRT-PCR. These studies were supported by NIH grant NS082203.

\section{Uncategorized References}

Bercury KK, Dai J, Sachs HH, Ahrendsen JT, Wood TL, Macklin WB. Conditional ablation of raptor or rictor has differential impact on oligodendrocyte differentiation and CNS myelination. $\mathrm{J}$ Neurosci. 2014; 34(13):4466-80. [PubMed: 24671993] 
Carracedo A, Baselga J, Pandolfi PP. Deconstructing feedback-signaling networks to improve anticancer therapy with mTORC1 inhibitors. Cell Cycle. 2008a; 7(24):3805-9. [PubMed: 19098454]

Carracedo A, Ma L, Teruya-Feldstein J, Rojo F, Salmena L, Alimonti A, Egia A, Sasaki AT, Thomas G, Kozma SC, et al. Inhibition of mTORC1 leads to MAPK pathway activation through a PI3Kdependent feedback loop in human cancer. J Clin Invest. 2008b; 118(9):3065-74. [PubMed: 18725988]

Chang A, Nishiyama A, Peterson J, Prineas J, Trapp BD. NG2-positive oligodendrocyte progenitor cells in adult human brain and multiple sclerosis lesions. J Neurosci. 2000; 20(17):6404-12. [PubMed: 10964946]

Chang A, Tourtellotte WW, Rudick R, Trapp BD. Premyelinating oligodendrocytes in chronic lesions of multiple sclerosis. N Engl J Med. 2002; 346(3):165-73. [PubMed: 11796850]

De Fea K, Roth RA. Modulation of insulin receptor substrate-1 tyrosine phosphorylation and function by mitogen-activated protein kinase. J Biol Chem. 1997; 272(50):31400-6. [PubMed: 9395471]

Delahaye L, Mothe-Satney I, Myers MG, White MF, Van Obberghen E. Interaction of insulin receptor substrate-1 (IRS-1) with phosphatidylinositol 3-kinase: effect of substitution of serine for alanine in potential IRS-1 serine phosphorylation sites. Endocrinology. 1998; 139(12):4911-9. [PubMed: 9832428]

Flores AI, Mallon BS, Matsui T, Ogawa W, Rosenzweig A, Okamoto T, Macklin WB. Akt-mediated survival of oligodendrocytes induced by neuregulins. J Neurosci. 2000; 20(20):7622-30. [PubMed: 11027222]

Flores AI, Narayanan SP, Morse EN, Shick HE, Yin X, Kidd G, Avila RL, Kirschner DA, Macklin WB. Constitutively active Akt induces enhanced myelination in the CNS. J Neurosci. 2008; 28(28): 7174-83. [PubMed: 18614687]

Francipane MG, Lagasse E. Selective targeting of human colon cancer stem-like cells by the mTOR inhibitor Torin-1. Oncotarget. 2013; 4(11):1948-62. [PubMed: 24185040]

Fyffe-Maricich SL, Karlo JC, Landreth GE, Miller RH. The ERK2 mitogen-activated protein kinase regulates the timing of oligodendrocyte differentiation. J Neurosci. 2011; 31(3):843-50. [PubMed: 21248107]

Guardiola-Diaz HM, Ishii A, Bansal R. Erk1/2 MAPK and mTOR signaling sequentially regulates progression through distinct stages of oligodendrocyte differentiation. Glia. 2012; 60(3):476-86. [PubMed: 22144101]

Gudz TI, Komuro H, Macklin WB. Glutamate stimulates oligodendrocyte progenitor migration mediated via an alphav integrin/myelin proteolipid protein complex. J Neurosci. 2006; 26(9): 2458-66. [PubMed: 16510724]

Harrington LS, Findlay GM, Lamb RF. Restraining PI3K: mTOR signalling goes back to the membrane. Trends Biochem Sci. 2005; 30(1):35-42. [PubMed: 15653324]

Hennen S, Wang H, Peters L, Merten N, Simon K, Spinrath A, Blattermann S, Akkari R, Schrage R, Schroder R, et al. Decoding signaling and function of the orphan $\mathrm{G}$ protein-coupled receptor GPR17 with a small-molecule agonist. Sci Signal. 2013; 6(298):ra93. [PubMed: 24150254]

Ishii A, Furusho M, Bansal R. Sustained activation of ERK1/2 MAPK in oligodendrocytes and schwann cells enhances myelin growth and stimulates oligodendrocyte progenitor expansion. J Neurosci. 2013; 33(1):175-86. [PubMed: 23283332]

Ishii A, Fyffe-Maricich SL, Furusho M, Miller RH, Bansal R. ERK1/ERK2 MAPK signaling is required to increase myelin thickness independent of oligodendrocyte differentiation and initiation of myelination. J Neurosci. 2012; 32(26):8855-64. [PubMed: 22745486]

Li J, DeFea K, Roth RA. Modulation of insulin receptor substrate-1 tyrosine phosphorylation by an Akt/phosphatidylinositol 3-kinase pathway. J Biol Chem. 1999; 274(14):9351-6. [PubMed: 10092613]

Liu Q, Chang JW, Wang J, Kang SA, Thoreen CC, Markhard A, Hur W, Zhang J, Sim T, Sabatini DM, et al. Discovery of 1-(4-(4-propionylpiperazin-1-yl)-3-(trifluoromethyl)phenyl)-9(quinolin-3-yl)benz o[h][1,6]naphthyridin-2(1H)-one as a highly potent, selective mammalian target of rapamycin (mTOR) inhibitor for the treatment of cancer. J Med Chem. 2010; 53(19): 7146-55. [PubMed: 20860370] 
Ma L, Chen Z, Erdjument-Bromage H, Tempst P, Pandolfi PP. Phosphorylation and functional inactivation of TSC2 by Erk implications for tuberous sclerosis and cancer pathogenesis. Cell. 2005; 121(2):179-93. [PubMed: 15851026]

Mallon BS, Shick HE, Kidd GJ, Macklin WB. Proteolipid promoter activity distinguishes two populations of NG2-positive cells throughout neonatal cortical development. J Neurosci. 2002; 22(3):876-85. [PubMed: 11826117]

McCarthy KD, de Vellis J. Preparation of separate astroglial and oligodendroglial cell cultures from rat cerebral tissue. J Cell Biol. 1980; 85(3):890-902. [PubMed: 6248568]

Mitew S, Hay CM, Peckham H, Xiao J, Koenning M, Emery B. Mechanisms regulating the development of oligodendrocytes and central nervous system myelin. Neuroscience. 2013

Mothe I, Van Obberghen E. Phosphorylation of insulin receptor substrate-1 on multiple serine residues, 612, 632, 662, and 731, modulates insulin action. J Biol Chem. 1996; 271(19):11222-7. [PubMed: 8626671]

Narayanan SP, Flores AI, Wang F, Macklin WB. Akt signals through the mammalian target of rapamycin pathway to regulate CNS myelination. J Neurosci. 2009; 29(21):6860-70. [PubMed: 19474313]

Niba ET, Nagaya H, Kanno T, Tsuchiya A, Gotoh A, Tabata C, Kuribayashi K, Nakano T, Nishizaki T. Crosstalk between PI3 kinase/PDK1/Akt/Rac1 and Ras/Raf/MEK/ERK pathways downstream PDGF receptor. Cell Physiol Biochem. 2013; 31(6):905-13. [PubMed: 23817184]

Pedraza CE, Monk R, Lei J, Hao Q, Macklin WB. Production, characterization, and efficient transfection of highly pure oligodendrocyte precursor cultures from mouse embryonic neural progenitors. Glia. 2008; 56(12):1339-52. [PubMed: 18512250]

Rodriguez-Viciana P, Warne PH, Dhand R, Vanhaesebroeck B, Gout I, Fry MJ, Waterfield MD, Downward J. Phosphatidylinositol-3-OH kinase as a direct target of Ras. Nature. 1994; 370(6490): 527-32. [PubMed: 8052307]

Sarbassov DD, Ali SM, Sengupta S, Sheen JH, Hsu PP, Bagley AF, Markhard AL, Sabatini DM. Prolonged rapamycin treatment inhibits mTORC2 assembly and Akt/PKB. Mol Cell. 2006; 22(2): 159-68. [PubMed: 16603397]

Silbereis JC, Nobuta H, Tsai HH, Heine VM, McKinsey GL, Meijer DH, Howard MA, Petryniak MA, Potter GB, Alberta JA, et al. Olig1 function is required to repress dlx1/2 and interneuron production in Mammalian brain. Neuron. 2014; 81(3):574-87. [PubMed: 24507192]

Thoreen CC, Sabatini DM. Rapamycin inhibits mTORC1, but not completely. Autophagy. 2009; 5(5): 725-6. [PubMed: 19395872]

Tokumoto YM, Durand B, Raff MC. An analysis of the early events when oligodendrocyte precursor cells are triggered to differentiate by thyroid hormone, retinoic acid, or PDGF withdrawal. Dev Biol. 1999; 213(2):327-39. [PubMed: 10479451]

Trapp BD, Nishiyama A, Cheng D, Macklin W. Differentiation and death of premyelinating oligodendrocytes in developing rodent brain. J Cell Biol. 1997; 137(2):459-68. [PubMed: 9128255]

Tyler WA, Gangoli N, Gokina P, Kim HA, Covey M, Levison SW, Wood TL. Activation of the mammalian target of rapamycin (mTOR) is essential for oligodendrocyte differentiation. J Neurosci. 2009; 29(19):6367-78. [PubMed: 19439614]

Volkers M, Konstandin MH, Doroudgar S, Toko H, Quijada P, Din S, Joyo A, Ornelas L, Samse K, Thuerauf DJ, et al. Mechanistic target of rapamycin complex 2 protects the heart from ischemic damage. Circulation. 2013; 128(19):2132-44. [PubMed: 24008870]

Wahl SE, McLane LE, Bercury KK, Macklin WB, Wood TL. Mammalian target of rapamycin promotes oligodendrocyte differentiation, initiation and extent of CNS myelination. J Neurosci. 2014; 34(13):4453-65. [PubMed: 24671992]

Wang C, Cigliano A, Delogu S, Armbruster J, Dombrowski F, Evert M, Chen X, Calvisi DF. Functional crosstalk between AKT/mTOR and Ras/MAPK pathways in hepatocarcinogenesis: implications for the treatment of human liver cancer. Cell Cycle. 2013; 12(13):1999-2010. [PubMed: 23759595] 


\section{Main points}

The Akt/mTOR and Erk 1/2 pathways interact to regulate oligodendrocyte lineage differentiation in vitro. Akt/mTOR inhibition induced upregulation of the Erk1/2 pathway, but not vice versa. Akt/mTOR inhibition in vivo also induced Erk1/2 signaling. 

vitro
A

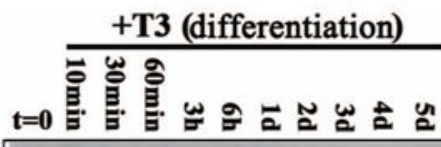

p-Akt S473
p-Akt T308
pan-Akt

p-Erk 1/2

t-Erk

p-mTOR

t-mTOR

p-S6 RP

GAPDH

p-IRS1 S318

p-IRS1 S307

p-IRS1 S612

IRS-1

MBP

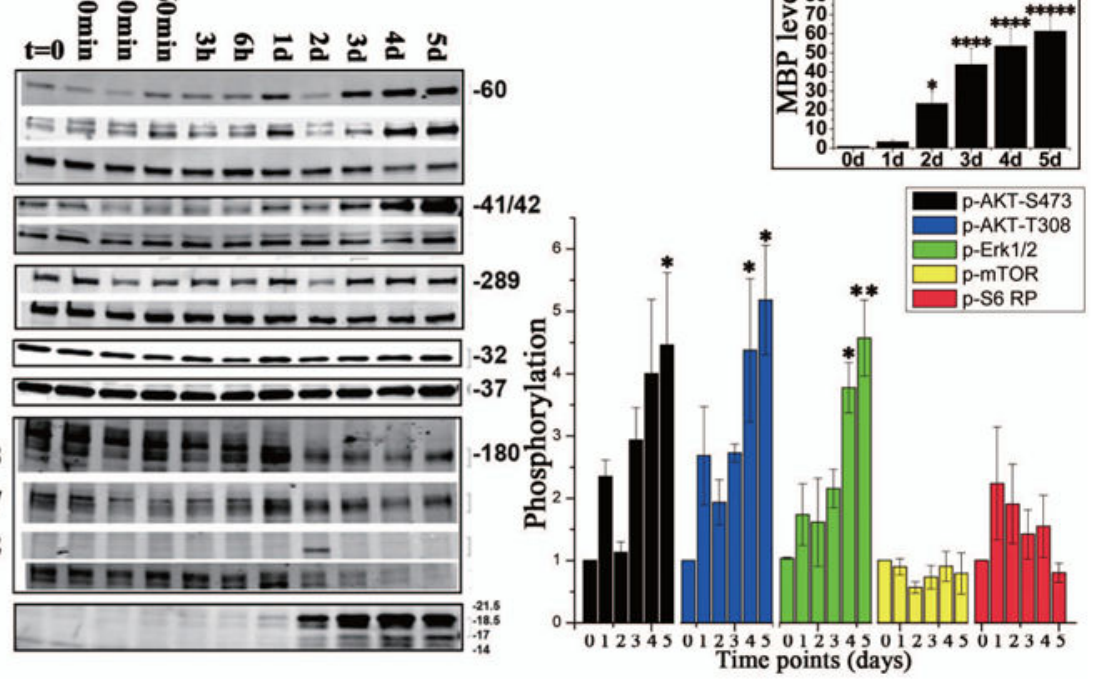

Figure 1. Activation of the Akt/mTOR and Erk 1/2 pathways increased as OPCs differentiated in

(A) Representative western blot analysis of components of the Akt/mTOR and Erk 1/2 pathways during oligodendrocyte differentiation. OPCs derived from rat mixed glia were treated with T3 for $10 \mathrm{~min}, 30 \mathrm{~min}, 60 \mathrm{~min}, 3 \mathrm{hr}, 6 \mathrm{hr}, 1 \mathrm{~d}, 2 \mathrm{~d}, 3 \mathrm{~d}, 4 \mathrm{~d}$, or 5d, collected and analyzed for phosphorylated or total Akt, p-mTOR S2448, S6RP, IRS1 (S318, S307, S612 and total) and phosphorylated or total Erk 1/2. MBP was quantified as a measure of oligodendrocyte differentiation, and GAPDH as the loading control. p-Akt S473 was increased while p-Akt T308 and total Akt showed little change. p-mTOR and p-S6RP expression remained constant as oligodendrocytes differentiated. p-IRS S318, S307 and total IRS1 decreased throughout differentiation, while pIRS1 S612 was only minimally detected. p-Erk1/2 decreased between 30 min and $6 \mathrm{~h}$ of T3 treatment, but increased from 1d until 5d; total Erk 1/2 expression remained consistent throughout differentiation. (B) Quantification of changes in phosphorylated states of Akt, Erk 1/2, mTOR and S6RP, measured as fold change relative to phosphorylation at day 0 . MBP expression also significantly increased from 1-5d post mitogen withdrawal (inset, top right graph). $\mathrm{n}=3$, error bars are graphed as SEM. *p $<0.05, * * \mathrm{p}<0.005, * * * \mathrm{p}<0.0005, * * * * \mathrm{p}<0.00005$ and $* * * * * \mathrm{p}<0.000005$ (One way ANOVA). 

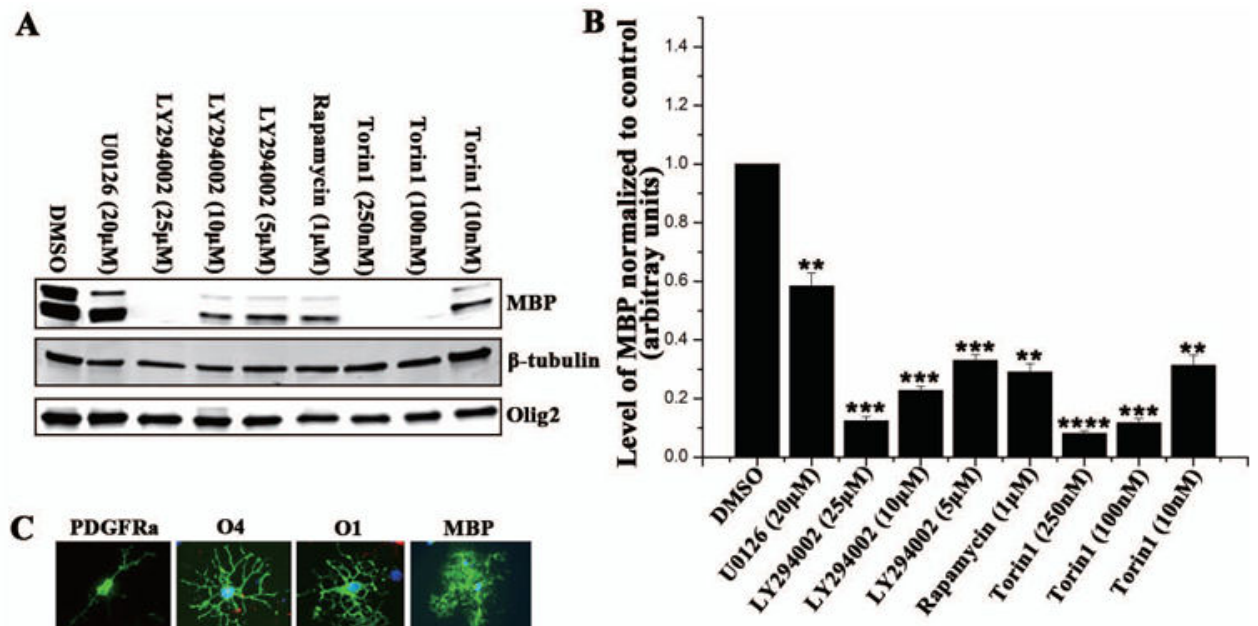

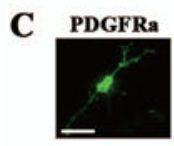

D
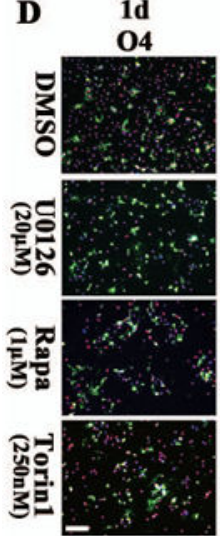

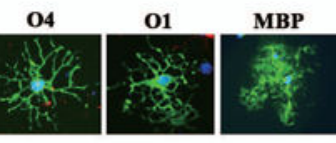

5d
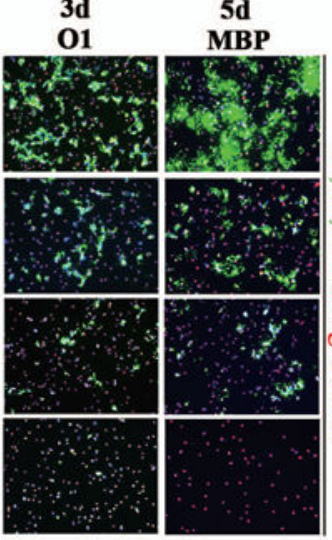
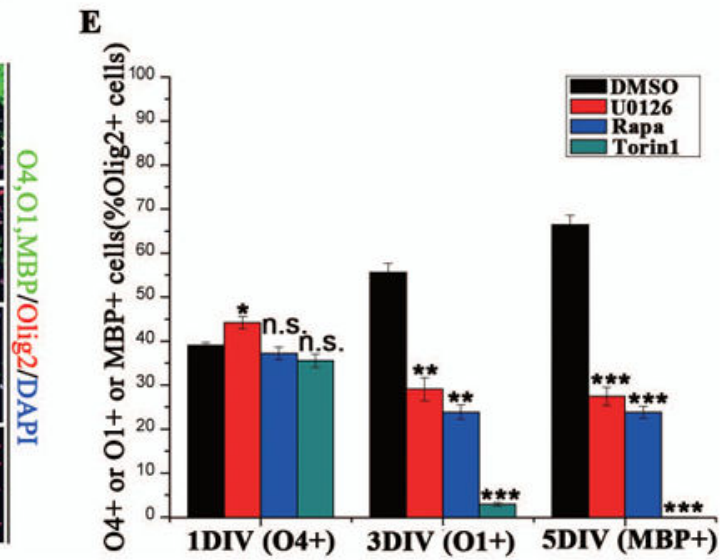

Figure 2. Pharmacological inhibition of Akt/mTOR or Erk1/2 decreased OPC differentiation in vitro

(A) Rat OPCs were incubated with pharmacological inhibitors of the Akt/mTOR and Erk $1 / 2$ pathways, and analyzed for MBP expression. Cells were treated with DMSO or specific inhibitors (U0126 [20 $\mu \mathrm{M}$ ], LY294002 [5 $\mu \mathrm{M}, 10 \mu \mathrm{M}$ or $25 \mu \mathrm{M}$ ], Rapamycin $[1 \mu \mathrm{M}$ ] and Torin1[10 nM, $100 \mathrm{nM}$ or $250 \mathrm{nM}]$ ) as they were switched to T3. Five days later, MBP expression was quantified as a measure of differentiation; $\beta$-tubulin and Olig2 were the general and the oligodendrocyte-specific loading controls, respectively. (B) Quantification of MBP protein expression normalized to a DMSO control. All inhibitor treatments resulted in a significant reduction in MBP expression. (C) Antigenic markers used to identify each stage of the OPC lineage during differentiation. (D) Rat OPCs were treated with either DMSO or specific inhibitors at induction of differentiation with T3. Cells were fixed after 1-, 3- or 5d of differentiation and labeled with O4, O1 or MBP (all in green). Olig2 (red) was used to label all oligodendrocyte lineage cells. (E) The percentage of co-labeled O4positive, O1-positive or MBP-positive/Olig2-positive cells (U0126:Red; Rapamycin: Purple; Torin 1:Green). Scale bars $=200 \mu \mathrm{m} . \mathrm{N}=3$ and graphed $+/-\operatorname{SEM}(\mathrm{B}, \mathrm{E}) . * \mathrm{p}<0.05, * * \mathrm{p}<$ $0.005, * * * \mathrm{p}<0.0005$ (one way ANOVA). 

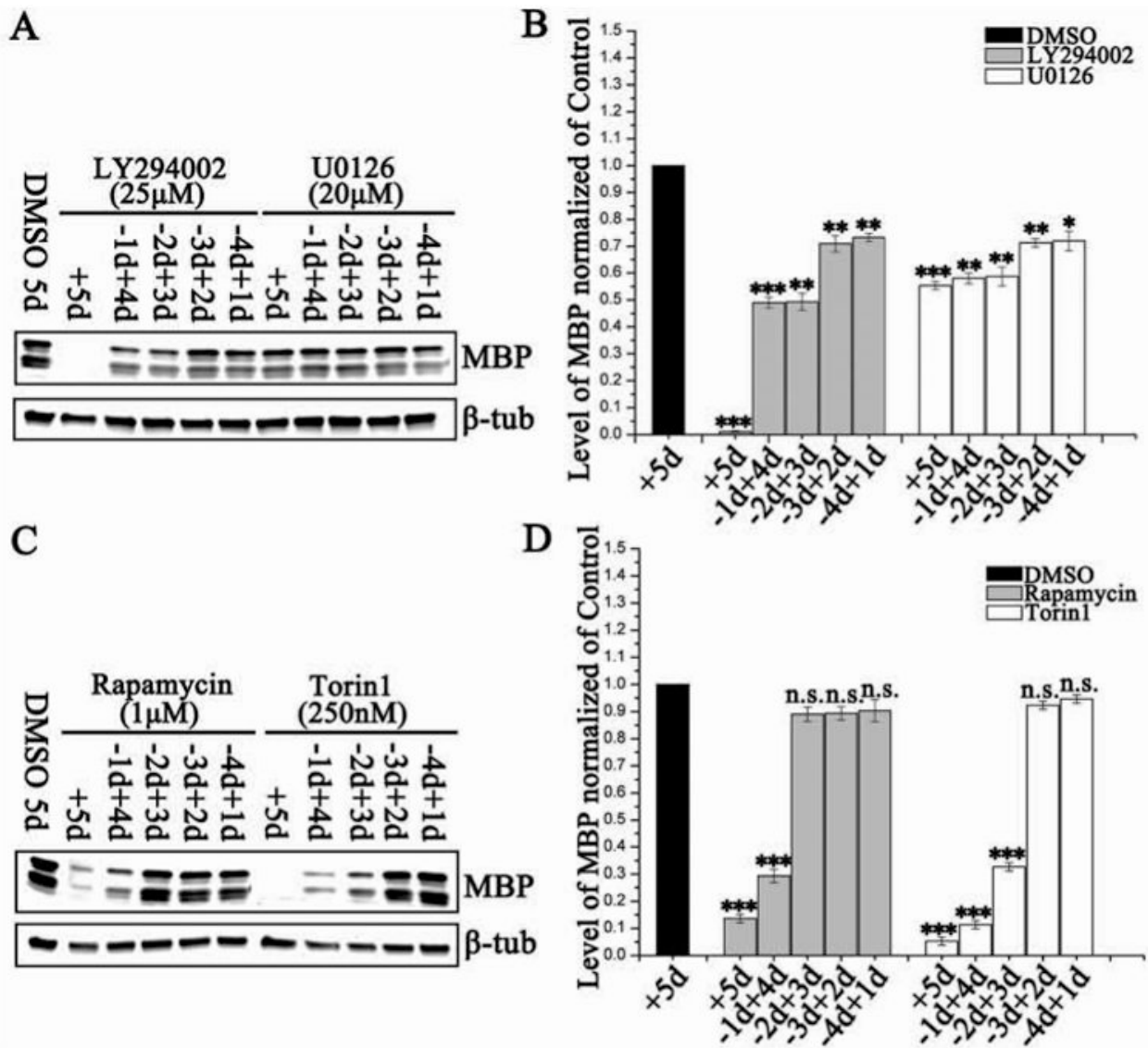

Figure 3. The Akt/mTOR and Erk 1/2 pathways regulate distinct stages of oligodendrocyte differentiation in vitro

Representative western blots of MBP expression in rat OPCs shifted to differentiation media (A) with or without LY294002 $(25 \mu \mathrm{M})$ or U0126 $(20 \mu \mathrm{M})$, or (C) with or without rapamycin $(1 \mathrm{uM})$ or Torin1 $(250 \mathrm{nM})$ over a 5 day time course. Drugs were added for the full time course $(+5 \mathrm{~d})$, after $1 \mathrm{~d}$ in T3 $(-1 \mathrm{~d}+4 \mathrm{~d})$, after $2 \mathrm{~d}$ in T3 $(-2 \mathrm{~d}+3 \mathrm{~d})$, after $3 \mathrm{~d}$ in T3 $(-3 \mathrm{~d}$ $+2 \mathrm{~d}$ ) or after $4 \mathrm{~d}$ in T3 $(-4 \mathrm{~d}+1 \mathrm{~d})$. $\beta$-tubulin was the loading control. (B, D) Quantification of western blot analysis for MBP protein with treatments (B:LY294002, gray bars; U0126, white bars; D:Rapamycin, gray bars; Torin1, white bars) standardized to a DMSO control. Data represent the mean \pm SEM from three independent experiments. $* \mathrm{p}<0.05$, $* * \mathrm{p}<$ $0.005, * * * \mathrm{p}<0.0005$ (One way ANOVA). 

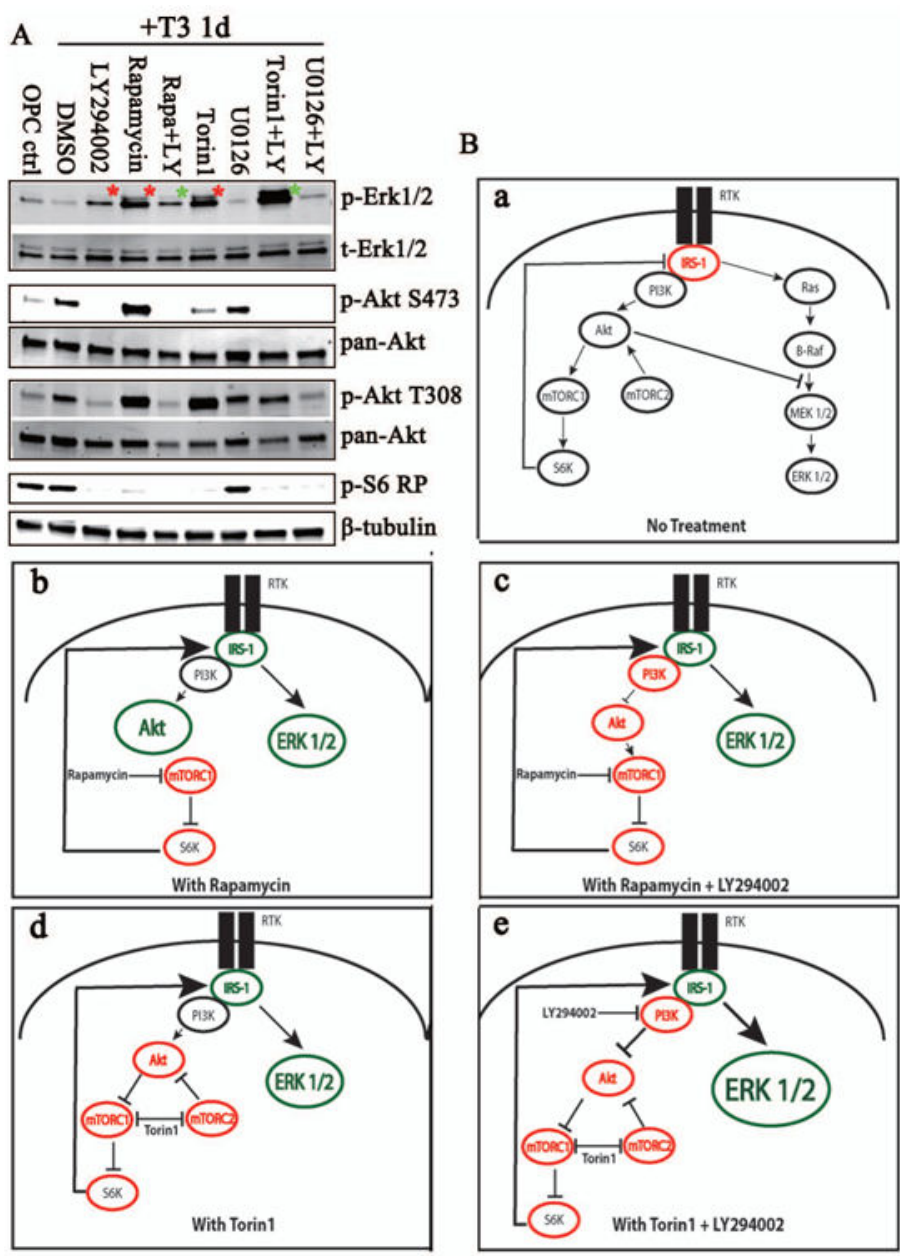

Figure 4. Inhibition of the Akt/mTOR or Erk 1/2 pathway did not result in bidirectional crosstalk

Single and combinatorial treatments of inhibitors were added to rat OPCs upon the addition of T3 and cells were collected 1 day post treatment. (A) Representative western blot analysis of phosphorylated and total Erk 1/2, Akt and S6RP expression levels. Increased pErk1/2 was observed after $24 \mathrm{~h}$ treatment with LY294002, Rapamycin or Torin1 (red asterisks).

Combined treatment with Rapamycin and LY294002 reduced Rapamycin-mediated Erk1/2 activation (green asterisk); in contrast, LY294002 and Torin1 increased the Torin1-mediated Erk pathway activation (green asterisk). (B) A schematic of the crosstalk between the pathways upon inhibitor treatments. Single or multiple drug treatments resulting in inhibited protein kinases (red) or activated protein kinases (green) are represented. Increased size of the kinase represents an increase in activation (larger circle $=$ dramatic increases) and the thick arrows represent a strong feedback mechanism (e.g., arrow from S6K to IRS-1). 

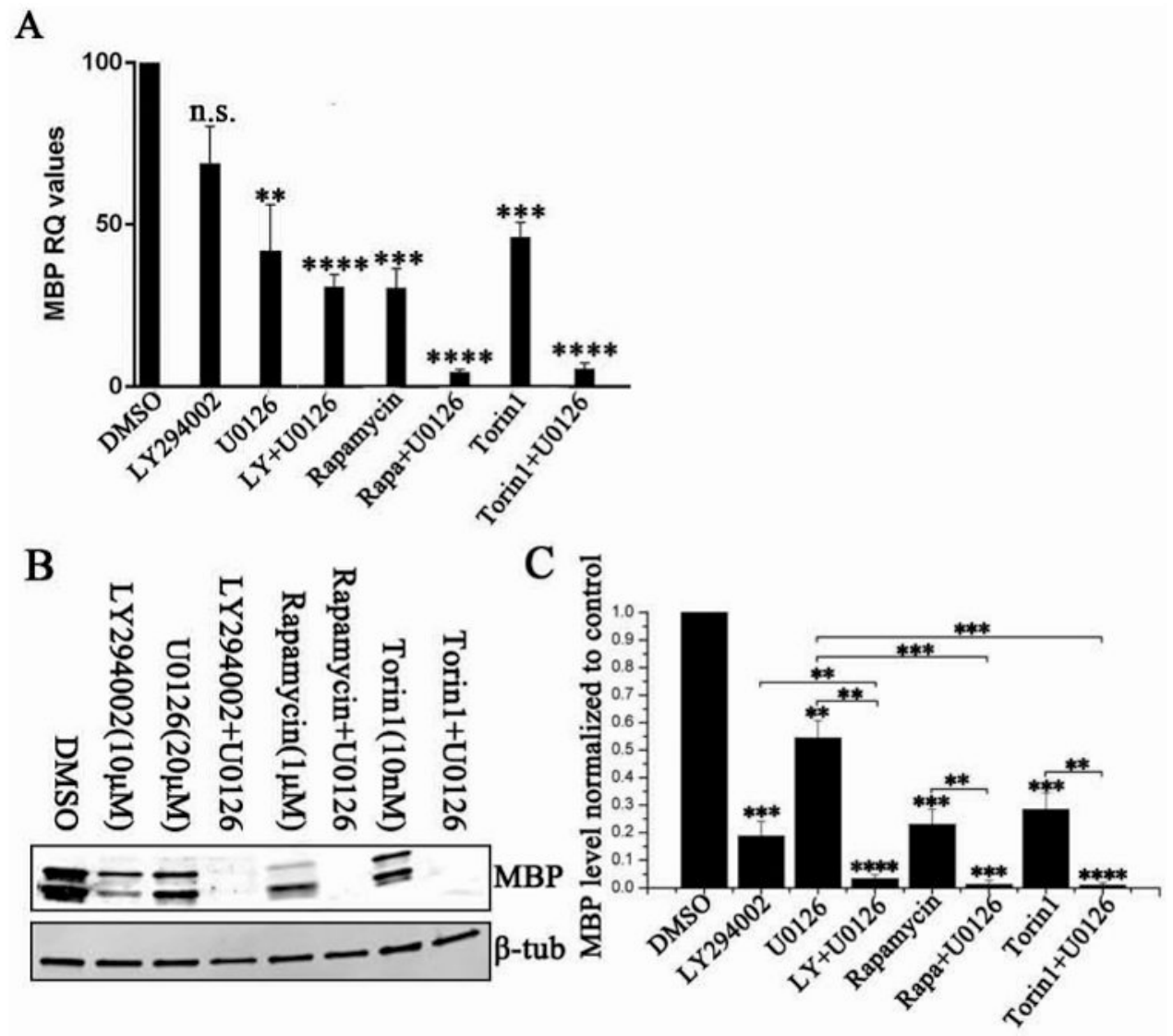

Figure 5. The Akt, mTOR and Erk 1/2 signaling pathways coordinately regulate both MBP mRNA and protein expression in vitro

Rat OPCs were treated with different kinase inhibitors, individually or in combination, and allowed to differentiate for 5 days. Cells were then collected for mRNA and protein analysis. (A) Combination treatments of U0126 and rapamycin, Torin1 or LY all resulted in greater reduction of MBP mRNA compared to single treatments. (B) Representative western blot analysis of MBP protein expression. (C) Quantification of MBP protein in treated cells. When correlated to the reduction in MBP mRNA, some combination treatments (treatments including LY294002; rapamycin or Torin1) had a significantly greater impact reducing MBP protein than MBP RNA. Data represent the mean \pm SEM from three independent experiments. $* \mathrm{p}<0.05, * * \mathrm{p}<0.005, * * * \mathrm{p}<0.0005, * * * * \mathrm{p}<0.00005$ (Two way ANOVA). 

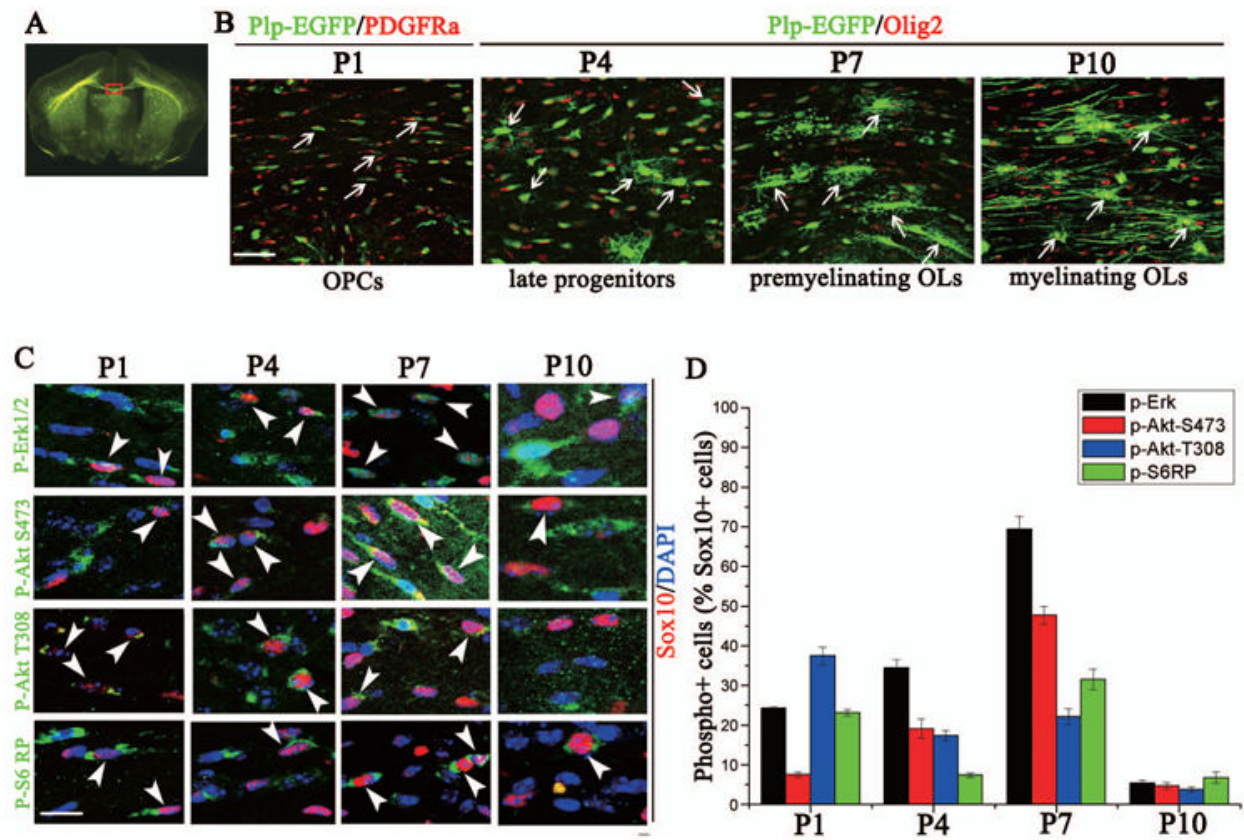

Figure 6. In vivo, Akt, mTOR and Erk 1/2 signaling are dynamically regulated during oligodendrocyte maturation from $\mathrm{P} 1$ to $\mathrm{P} 10$

(A) Representative PLP-EGFP coronal brain section outlining the area of interest (red). (B) Representative images at the midline of the PLP-EGFP corpus callosum with EGFP-positive oligodendrocyte lineage cells (green) co-stained with PDGFRa (red) for P1 section or Olig2 (red) for P4, P7 or P10 section. Arrows represent OPCs (P1 and P4), premyelinating oligodendrocytes (P7) or myelinating oligodendrocytes (P10). (C) Immunostaining of P1, P4, P7 and P10 mouse brains with p-Erk1/2, p-mTOR, p-Akt S473, p-Akt T308 and p-S6RP (green), Sox10 (red) and DAPI (blue). Arrowheads (white) identify specific oligodendrocytes co-labeled with the phosphorylated protein. (D) Quantification of the percentage of phosphorylated protein-positive cells/Sox10-positive cell. pErk 1/2 (black), pAkt 473 (red) and pS6RP (green) expression peaked in oligodendrocytes at P7, which correlates to the transition of OPCs to premyelinating oligodendrocytes (B), while pAkt 308 peaks earlier. Scale bars $=50 \mu \mathrm{m}(\mathrm{B})$ and $20 \mu \mathrm{M}(\mathrm{C})$. Data represents the mean $+/-$ SEM for three independent experiments. 

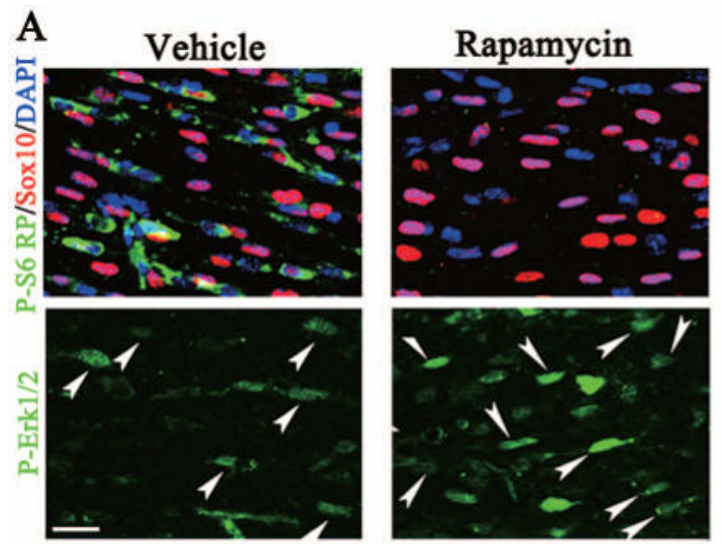

B.

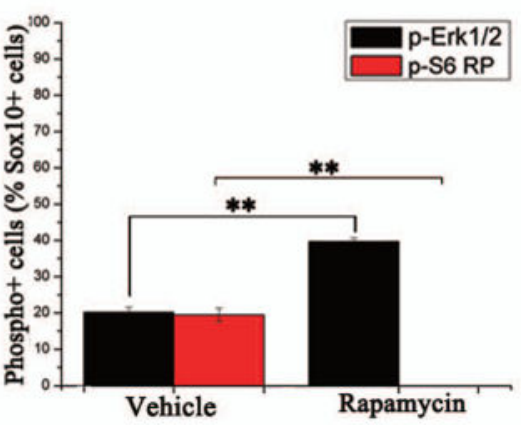

Figure 7. Pharmacological inhibition of $\mathrm{mTORC1}$ in vivo elevated the level of phosphorylated Erk1/2 signaling in both oligodendrocytes and neurons

Mice were injected IP at P7 with either vehicle or rapamycin, and tissue for immunohistochemistry was collected 24 hours post injection. (A) Representative images of the lateral corpus callosum of oligodendrocytes stained with pS6RP or pErk 1/2 (green), Sox10 (red) and DAPI (blue) of the vehicle (left panel) or rapamycin injected animals (right). Effective inhibition of mTORC1 was seen by complete loss of pS6RP detection in the rapamycin injected animals (top, right). Arrows represent pErk $1 / 2+/$ Sox $10+$ cells. (B) Quantification of oligodendrocyte co-expressing pErk 1/2 (black) or pS6RP (red)/Sox10 positive cells in vehicle-injected compared to rapamycin-injected animals. Note that there is essentially complete loss of pS6RP in rapamycin-injected animals (i.e., absence of far right column). Scale bars $=20 \mu \mathrm{M}$. $* * \mathrm{p}<0.05$ (Student's t test). 